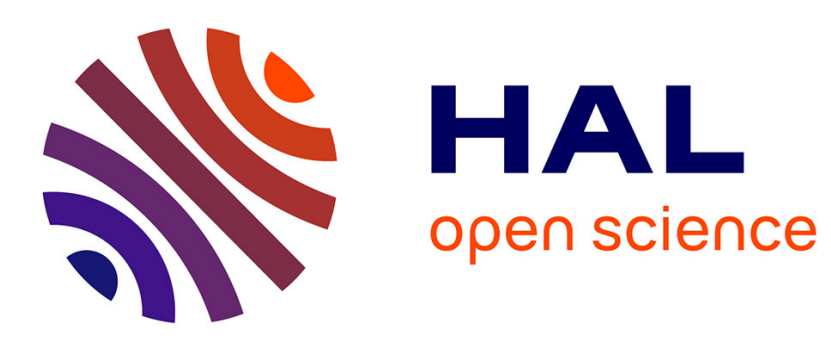

\title{
Dynamics of a parallel-plate electrostatic actuator in viscous dielectric media
}

Sindhu Preetham Burugupally, W. Roshantha Perera

\section{To cite this version:}

Sindhu Preetham Burugupally, W. Roshantha Perera. Dynamics of a parallel-plate electrostatic actuator in viscous dielectric media. Sensors and Actuators A: Physical , 2019, 295, pp.366-373. 10.1016/j.sna.2019.06.005 . hal-02269570

\section{HAL Id: hal-02269570 \\ https://hal.science/hal-02269570}

Submitted on 23 Aug 2019

HAL is a multi-disciplinary open access archive for the deposit and dissemination of scientific research documents, whether they are published or not. The documents may come from teaching and research institutions in France or abroad, or from public or private research centers.
L'archive ouverte pluridisciplinaire HAL, est destinée au dépôt et à la diffusion de documents scientifiques de niveau recherche, publiés ou non, émanant des établissements d'enseignement et de recherche français ou étrangers, des laboratoires publics ou privés. 


\title{
Dynamics of a parallel plate electrostatic actuator in viscous dielectric media
}

\author{
Sindhu Preetham Burugupally ${ }^{\mathrm{a}, 1, *}$ W. Roshantha Perera ${ }^{\mathrm{a}, 1}$ \\ ${ }^{a}$ Department of Mechanical Engineering, Wichita State University, Wichita, KS 67260 USA
}

\begin{abstract}
Understanding the dynamics of a parallel plate electrostatic actuator in viscous dielectric media will help optimize the actuator performance for manipulating microparticles suspended in aqueous media. In this paper, we analyze the response of the actuator in a clamped-clamped configuration immersed in viscous dielectric media. We modeled the actuator as a continuous system by deriving a reduced-order model, and solving it by employing the Galerkin method and linear undamped mode shapes for a clamped-clamped beam. Our model incorporates the inertial loading effect and squeeze film damping by the media, nonlinear mid-plane stretching forces in the beam electrode of the actuator, and nonlinear contact force during the physical contact of the beam electrode with the stationary electrode. The model is utilized to study the actuator dynamics over a broad range, three orders of magnitude of viscosity and two orders of magnitude of relative permittivity of the media. We report that the actuator at lower actuation voltages and/or higher actuation frequencies can be approximated to be a linear system. We also report that at very high actuation frequencies, the beam electrode does not pull-in into the stationary electrode, and the actuator experiences a significant phase lag and displacement drift over time.
\end{abstract}

Keywords: Electrostatic actuator, viscous media, reduced order modeling, mechanics.

\section{Introduction}

Electrostatic microactuators are commonly employed for generating forces on the order of few microNewtons and displacements on the order of few microns for a wide range of manipulating and probing applications. Applications include aligning the optical fibers in optoelectronic packaging[1, handling of micron-sized objects[2], probing of biological cells 3], actuating the miniature fins on millimeterscale rockets 4, and switching the radio frequency

\footnotetext{
* Corresponding author Email address:

sindhupreetham.burugupally@wichita.edu (Sindhu

Preetham Burugupally)

${ }^{1}$ equal contribution
}

signals [5]. Parallel plate actuators are a class of electrostatic actuators known for high displacement and force resolutions 6 . It is difficult to predict the dynamics of these actuators as they have a nonlinear dynamic response due to the nonlinear squeeze film damping, nonlinear internal stretching, and nonlinear electrostatic actuation forces 7] (Fig. 1). Understanding the dynamics of a parallel plate actuator will help optimize actuator performance for a specific application such as manipulating biological cells. To understand the static and dynamic response of these actuators, researchers modeled them either as lumped systems 8 or continuous systems 9 , 10. Most efforts were directed towards the design of actuators operating in air or vacuum where the fluid viscous damping is negligible or absent [11, 12, 13. 

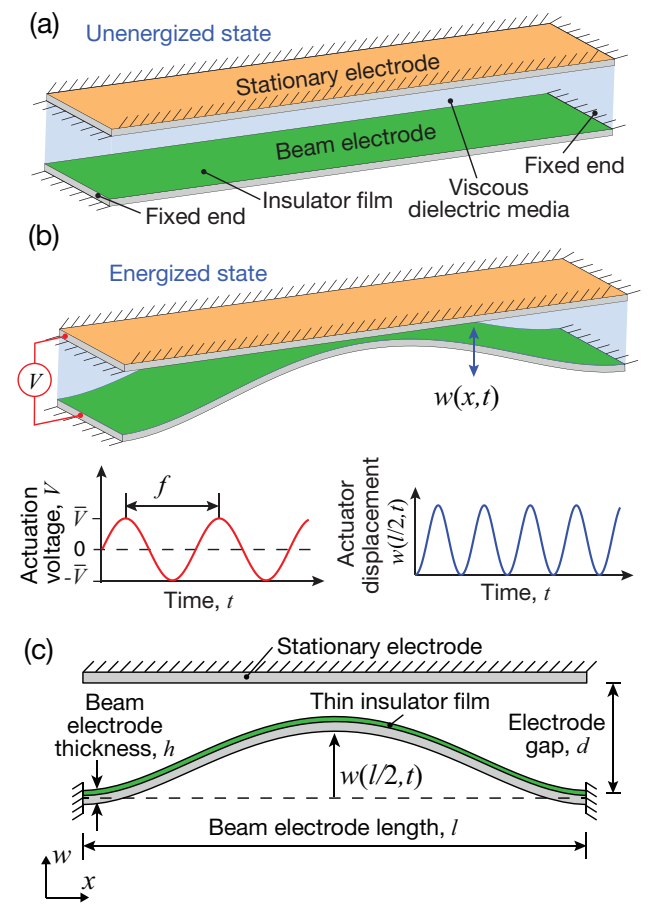

Figure 1: Schematic sketch of the parallel plate electrostatic actuator. (a) Unenergized state. (b) Energized state. (c) A side view of the actuator in the energized stage with parameters shown.

With the recent interest in manipulating and probing of biological cells on lab-on-a-chip platform, different class of actuators - comb drives 3 , curved electrodes [14, and hybrid drives [15] - are being designed to operate in viscous dielectric (aqueous) media. The performance of these actuators characterized in terms of actuator displacement and frequency response depends on the relative permittivity and dynamic viscosity of the viscous dielectric media [16, 17]. The high relative permittivity of aqueous media $\left(\epsilon_{f, w}=80\right)$ amplifies the generated force and displacement, while the high dynamic viscosity $\left(\mu_{w}=8.9 \times 10^{-4} \mathrm{~Pa}-\mathrm{s}\right)$ restricts the displacement and narrows the bandwidth frequency of the actuator 16 - hence it is important to understand the effect of media properties on the actuator performance. Some of the recent research efforts include: investigating the effect of fluid viscosity, initial electrode gap, and beam geometry [18; determining an analytical expression for pull-in time of actuator [19]; deriving physics-based models to predict the actuator static performance 20; optimizing actuator design parmeters to achieve large displacements in aqueous media[15]; and identifying means to extend the displacement range of the actuator in liquid solutions 8 .

Table 1: Actuator parameters and physical properties.

\begin{tabular}{ll}
\hline Physical parameter (units) & Value \\
\hline Length of the beam electrode, $l(\mu \mathrm{m})$ & 2000 \\
Thickness of the beam electrode, $h(\mu \mathrm{m})$ & 5 \\
Width of the beam electrode, $b(\mu \mathrm{m})$ & 45 \\
Gap between the electrodes, $d(\mu \mathrm{m})$ & 5 \\
$\begin{array}{l}\text { Density of the electrode material, }, \rho \\
\left(\mathrm{kg} / \mathrm{m}^{3}\right)\end{array}$ & 2329 \\
Young's Modulus, $E$ (GPa) & 130 \\
Axial tensile force/unit length, $\hat{N}(\mathrm{~N} / \mathrm{m})$ & 0 \\
Relative permittivity of air, $\epsilon_{f, a}$ & 1 \\
Relative permittivity of methanol, $\epsilon_{f, m}$ & 32 \\
Relative permittivity of water, $\epsilon_{f, w}$ & 80 \\
Dynamic viscosity of air, $\mu_{a}(\mu \mathrm{Pa}-\mathrm{s})$ & 18.1 \\
$\begin{array}{l}\text { Dynamic viscosity of methanol, } \mu_{m}(\mu \mathrm{Pa}-\mathrm{s}) \\
\text { Dynamic viscosity of water, } \mu_{w}(\mu \mathrm{Pa}-\mathrm{s})\end{array}$ & 700 \\
Nondimensional damping term $c$ for air, $c_{a}$ & 10 \\
Nondimensional damping term $c$ for & 120 \\
methanol, $c_{m}$ & \\
Nondimensional damping term $c$ for water, & 200 \\
$c_{w}$ & 1.2 \\
Density of air, $\rho_{f, a}\left(\mathrm{~kg} / \mathrm{m}^{3}\right)$ & 790 \\
Density of methanol, $\rho_{f, m}\left(\mathrm{~kg} / \mathrm{m}^{3}\right)$ & 1000 \\
Density of water, $\rho_{f, w}\left(\mathrm{~kg} / \mathrm{m}^{3}\right)$ & \\
\hline
\end{tabular}

In this paper, we analyze the dynamics of a parallel plate actuator in a clamped-clamped configuration immersed in viscous dielectric media (Fig. 1). Here, we model the actuator as a continuous system by deriving a reduced-order model similar to the work presented in Ref. 21]. The model is solved by employ- 
ing the Galerkin method 22] and linear undamped mode shapes for a clamped-clamped beam 23. The work presented here extends the reduced-order model framework used by Younis et al. [9] by modeling actuator contact physics to describe the actuator dynamics beyond the pull-in instability. Our model incorporates the inertial loading effect and squeeze film damping by the media, nonlinear mid-plane stretching forces in the beam electrode of the actuator, and nonlinear contact force 24 during the physical contact of the beam electrode with the stationary electrode (Fig. 1). The model is first validated using the data available in the existing literature 9 and then the model is utilized to study the actuator dynamics over a broad range, three orders of magnitude of viscosity (relates to damping or $Q$-factor) and two orders of magnitude of relative permittivity of the media. We describe the actuator characteristics in terms of pull-in time, and actuator displacement for both $\mathrm{DC}$ and $\mathrm{AC}$ actuation voltages. The following sections describe the design, model, and results that describe the response of the actuator in different viscous dielectric media.

\section{Design}

The actuator is designed to achieve $1-5 \mu \mathrm{m}$ displacement range and operate in viscous dielectric media for low actuation voltages $(<20 \mathrm{~V})$. The actuator comprises of two main components: a beam electrode to deliver displacement strokes and a stationary electrode to drive the beam electrode in the forward direction (Fig. 1 (a)). The beam electrode is a clamped-clamped structure that is compliant, while the stationary electrode is an immovable rigid member. The actuator motion can result in axial stretching of the beam electrode. The electrodes are coated with an insulator film for electrical insulation. The nominal actuator parameters and physical properties of media are listed in Table 1. Upon energizing the actuator by supplying voltage $V$, the beam electrode moves in-plane closer to the stationary electrode (Fig. 1(b,c)). The actuation voltage $V$ is given by the function $V(t)=\bar{V} \sin 2 \pi f t$, where $\bar{V}$ is the voltage amplitude, $f$ is the frequency of the actuation voltage, and $t$ is the time. Note that for operating the actuator in ionic viscous media (water), a pure sinusoidal voltage function can result in electrolysis, anodization, and electrode polarization [25]. Hence, the actuator will be supplied with an amplitude modulated actuation signal given by the function $V(t)=$ $\frac{1}{2} \bar{V}(1+\cos 2 \pi f t) \Pi(t)$ where $\bar{V}$ is the voltage amplitude, $\cos 2 \pi f t$ is the modulation waveform, $t$ is the time, $\Pi(t)=\frac{4}{\pi} \sum_{j=1}^{\infty} \frac{1}{2 j-1}(-1)^{j-1} \cos 2 \pi f_{\mathrm{c}} j t$ is a high-frequency, zero-mean pulse signal to prevent charge shielding effects [25], and $f$ and $f_{c}=500 \mathrm{kHz}$ are the modulation and carrier frequencies, respectively. However, for uniformity, in this work we are restricting the actuation voltage to be a pure sinusoidal function.

\section{Model}

The actuator shown in Fig. 1 is modeled as a continuous system by deriving a reduced-order model and solved by employing the Galerkin method and linear undamped mode shapes for a clamped-clamped beam. The model incorporates the inertial loading effect and squeeze film damping by the media, nonlinear mid-plane stretching forces in the beam electrode of the actuator, and nonlinear contact force during the physical contact of the beam electrode with the stationary electrode (Fig. 11). The actuator motion is described by Eq. 1a with boundary conditions given by Eqs. $1 b$ and $1 \mathrm{c}$.

$$
\begin{gathered}
E I \frac{\partial^{4} w}{\partial x^{4}}+\left(\rho b h+0.785 \rho_{f} b^{2}\right) \frac{\partial^{2} w}{\partial t^{2}}+\hat{c} \frac{\partial w}{\partial t} \\
=\left[\frac{E A}{2 l} \int_{0}^{l}\left(\frac{\partial w}{\partial x}\right)^{2} \mathrm{~d} x+\hat{N}\right] \frac{\partial^{2} w}{\partial x^{2}}+\frac{\epsilon_{0} \epsilon_{f} b V^{2}}{2(d-w)^{2}} \\
-k_{c} g^{3}\left(1+\hat{\mu_{c}} \frac{\partial g}{\partial t}\right) H \\
\quad(1 \mathrm{a}) \\
\frac{\partial w}{\partial x}(0, t)=\frac{\partial w}{\partial x}(l, t)=0,
\end{gathered}
$$

where $w$ is the deflection of the beam electrode, $x$ is the coordinate along the beam electrode, $b$ is the width of the beam electrode, $h$ is the thickness of 
the beam electrode, $l$ is the length of the beam electrode, $d$ is the gap between the beam electrode and the stationary electrode, $A$ is the cross-sectional area of the beam electrode, $E$ is the Young's modulus of the beam electrode material, $I=b h^{3} / 12$ is the area moment of inertia of the beam electrode, $t$ is the time, $\rho$ is the density of the beam electrode material, $\rho_{f}$ is the density of the media, $\epsilon_{0}$ is the permittivity of vacuum, $\epsilon_{f}$ is relative permittivity of the media, $\hat{c}$ is the damping coefficient by the media per unit length of the beam electrode, $\hat{N}$ is the axial tensile force in the beam electrode per unit length of the beam electrode, $V$ is the actuation voltage at time $t, g=(d-w)$ is the gap between the beam electrode and stationary electrode at time $t, H$ is the Heaviside step function in terms of $g$, and $\hat{\mu}_{c}=0.5 \mathrm{~s} / \mathrm{m}$ models the damping and $k_{c}=10^{15} \mathrm{~N} / \mathrm{m}^{4}$ models nonlinear spring stiffness during the contact of the beam electrode with the stationary electrode, respectively. Note that damping term $\hat{c}$ for each media is calculated using the squeeze film damping model presented in Ref. [26, and the numerical values of $\hat{\mu}_{c}$ and $k_{c}$ are based on the work by Vyasarayani et al. 24. Also, note that the effect of insulator film on the actuator dynamics is not considered in this work.

For analysis, the model (Eqs. $1 \mathrm{a} / \mathrm{c}$ is nondimensionalized by applying the following scales to the variables:

$$
W=\frac{w}{d}, \quad X=\frac{x}{l}, \quad T=\frac{t}{t_{p}}, \quad G=\frac{g}{d}
$$

where $t_{p}=\sqrt{\frac{\rho b h l^{4}}{E I}}$ is the reference time period. After applying these scales, the model (Eqs. 1a 1c becomes

$$
\begin{gathered}
\frac{\partial^{4} W}{\partial X^{4}}+m \frac{\partial^{2} W}{\partial T^{2}}+c \frac{\partial W}{\partial T} \\
=\left[\alpha_{1} \Gamma(W, W)+N\right] \frac{\partial^{2} W}{\partial X^{2}}+\frac{\alpha_{2} V^{2}}{(1-W)^{2}} \\
\quad-\alpha_{3}(1-W)^{3}\left(1-\mu_{c} \frac{\partial W}{\partial T}\right) H \\
W(0, T)=W(1, T)=0
\end{gathered}
$$

$$
\frac{\partial W}{\partial X}(0, T)=\frac{\partial W}{\partial X}(1, T)=0
$$

where

$$
\begin{gathered}
m=\left(1+\frac{0.785 \rho_{f} b}{\rho h}\right), \quad c=\frac{\hat{c} l^{4}}{E I t_{p}}, \quad \alpha_{1}=\frac{6 d^{2}}{h^{2}} \\
\Gamma_{1,2}=\Gamma\left(\psi_{1}, \psi_{2}\right)=\int_{0}^{1} \frac{\partial \psi_{1}}{\partial X} \frac{\partial \psi_{2}}{\partial X} \mathrm{~d} X, \quad N=\frac{\hat{N} l^{2}}{E I} \\
\alpha_{2}=\frac{6 \epsilon_{0} \epsilon_{f} l^{4}}{E h^{3} d^{3}}, \quad \alpha_{3}=\frac{k_{c} d^{2} L^{4}}{E I}, \quad \mu_{c}=\frac{\hat{\mu}_{c} d}{t_{p}}
\end{gathered}
$$

To characterize the actuator dynamics, the model (Eqs. 3a 3c is solved numerically using MATLAB. For this, Eq. 3a is rearranged by multiplying it with $(1-W)^{2}$. Then, the variable $W($ or $W(X, T))$ is expressed as a summation of product of temporal $U_{i}(T)$ and spatial $\phi_{i}(X)$ variables, that is, $W(X, T)=\sum_{i=1}^{2 M-1} U_{i}(T) \phi_{i}(X)$. Here, $i$ is the mode number, $\phi_{i}(X)$ is the $i^{\text {th }}$ mode shape of an undamped clamped-clamped beam with negligible axial loads $(\hat{N}=0)[23$, and $M$ is the total number of mode shapes and $2 M-1$ is the highest mode shape used to represent the beam electrode displacement, respectively. Note that only symmetric mode shapes $(i \in\{1,3, \ldots 2 M-1\})$ are used in this analysis. The model is simplified by invoking the normal mode shape property $\int_{0}^{1} \phi_{i} \phi_{j} \mathrm{~d} X=\delta_{i j}\left[22\right.$, where $\delta_{i j}$ is the Kronecker delta function. The simplified model equation becomes

$$
\begin{aligned}
& m\left(\ddot{U}_{n}-2 \sum_{i, j=1}^{2 M-1} \ddot{U}_{i} U_{j} \int_{0}^{1} \phi_{i} \phi_{j} \phi_{n} \mathrm{~d} X\right. \\
& \left.+\sum_{i, j, k=1}^{2 M-1} \ddot{U}_{i} U_{j} U_{k} \int_{0}^{1} \phi_{i} \phi_{j} \phi_{k} \phi_{n} \mathrm{~d} X\right)+c \dot{U}_{n}+\omega_{n}^{2} U_{n} \\
& =\alpha_{1} \sum_{i, j, k=1}^{2 M-1} U_{i} U_{j} U_{k} \Gamma_{i, j} \int_{0}^{1} \phi_{k}^{\prime \prime} \phi_{n} \mathrm{~d} X \\
& +\alpha_{1} \sum_{i, j, k, l, m=1}^{2 M-1} U_{i} U_{j} U_{k} U_{l} U_{m} \Gamma_{i, j} \int_{0}^{1} \phi_{k} \phi_{l} \phi_{m}^{\prime \prime} \phi_{n} \mathrm{~d} X \\
& -2 \alpha_{1} \sum_{i, j, k, l=1}^{2 M-1} U_{i} U_{j} U_{k} U_{l} \Gamma_{i, j} \int_{0}^{1} \phi_{k} \phi_{l}^{\prime \prime} \phi_{n} \mathrm{~d} X
\end{aligned}
$$




$$
\begin{aligned}
& +\alpha_{2} V^{2} \int_{0}^{1} \phi_{n} \mathrm{~d} X \\
& -\alpha_{3} H \int_{0}^{1} \phi_{n}\left(1-\sum_{i=1}^{2 M-1} U_{i} \phi_{i}\right)^{3}\left(1-\mu_{c} \sum_{i=1}^{2 M-1} \dot{U}_{i} \phi_{i}\right) \mathrm{d} X \\
& +2 \sum_{i, j=1}^{2 M-1} \omega_{i}^{2} U_{i} U_{j} \int_{0}^{1} \phi_{i} \phi_{j} \phi_{n} \mathrm{~d} X \\
& -\sum_{i, j, k=1}^{2 M-1} \omega_{i}^{2} U_{i} U_{j} U_{k} \int_{0}^{1} \phi_{i} \phi_{j} \phi_{k} \phi_{n} \mathrm{~d} X \\
& +N \sum_{i=1}^{2 M-1} U_{i}^{1} \int_{0}^{\prime \prime} \phi_{n} \mathrm{~d} X \\
& +N \sum_{i, j, k=1}^{2 M-1} U_{i} U_{j} U_{k} \int_{0}^{1} \phi_{i} \phi_{j} \phi_{k}^{\prime \prime} \phi_{n} \mathrm{~d} X \\
& -2 N \sum_{i, j=1}^{2 M-1} U_{i} U_{j} \int_{0}^{1} \phi_{i} \phi_{j}^{\prime \prime} \phi_{n} \mathrm{~d} X \\
& +2 c \sum_{i, j=1}^{2 M-1} \dot{U}_{i} U_{j} \int_{0}^{1} \phi_{i} \phi_{j} \phi_{n} \mathrm{~d} X \\
& \sum_{i, k=1}^{2 M-1} \dot{U}_{i} U_{j} U_{k} \int_{0}^{1} \phi_{i} \phi_{j} \phi_{k} \phi_{n} \mathrm{~d} X
\end{aligned}
$$

where $n \in\{1,3,5, \ldots 2 M-1\}$, and overdot ' ' and prime ' ' ' correspond to temporal and spatial derivatives, respectively. Eq. 5 represents a system of $M$ nonlinear ordinary differential equations; the system of equations is solved numerically to obtain the numerical values of the temporal variable: $U_{1}, U_{3}, U_{5}, \ldots$, and $U_{2 M-1}$.

For static analysis, the time derivative terms in Eq. 5 are dropped, yielding a system of $M$ polynomial equations. The polynomial equation set is solved for $U$ 's comprising of $U_{1}, U_{3}, \ldots U_{2 M-1}$ for a given DC actuation voltage $\bar{V}$ using the built-in MATLAB function fsolve. For each DC actuation voltage, a $U_{i}$ has two possible solutions - one results in a stable configuration and other in a unstable configuration of the beam electrode. The corresponding actuator displacement amplitudes $W(1 / 2)$ can be computed using the aforementioned equation $W(X=1 / 2, T)=\sum_{i=1}^{2 M-1} U_{i}(T) \phi_{i}(X=1 / 2)$. For dynamic analysis, Eq. 5 is solved for $U$ 's comprising of $U_{1}, U_{3}, \ldots U_{2 M-1}$ for a given actuation voltage $V$ using the built-in MATLAB function ode15s for a given set of initial conditions: nondimensional actuator displacement $W(X, 0)=0$ and nondimensional actuator velocity $\dot{W}(X, 0)=0$. The actuator displacement $W(X, T)$ at nondimensional time $T$, can be computed using the aforementioned equation $W(X, T)=\sum_{i=1}^{2 M-1} U_{i}(T) \phi_{i}(X)$

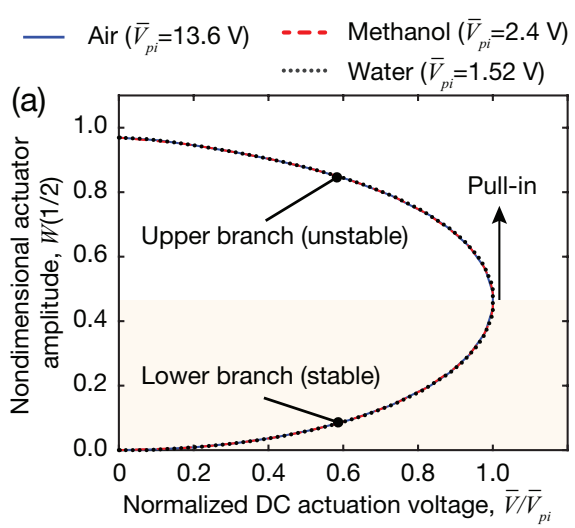

(b)

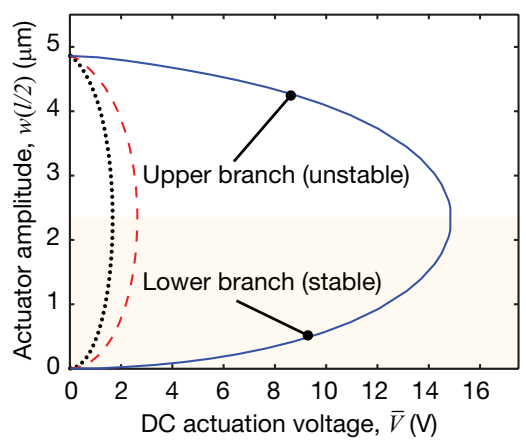

Figure 2: Static equilibrium curves for the actuator in air, methanol, and water media. Curves plotted for all the media against (a) normalized DC actuation voltage $\bar{V} / \bar{V}_{p i}$ with nondimensional actuator displacement amplitude $W(1 / 2)$ (b) DC actuation voltage $\bar{V}$ with dimensional actuator displacement amplitude $w(l / 2)$. 

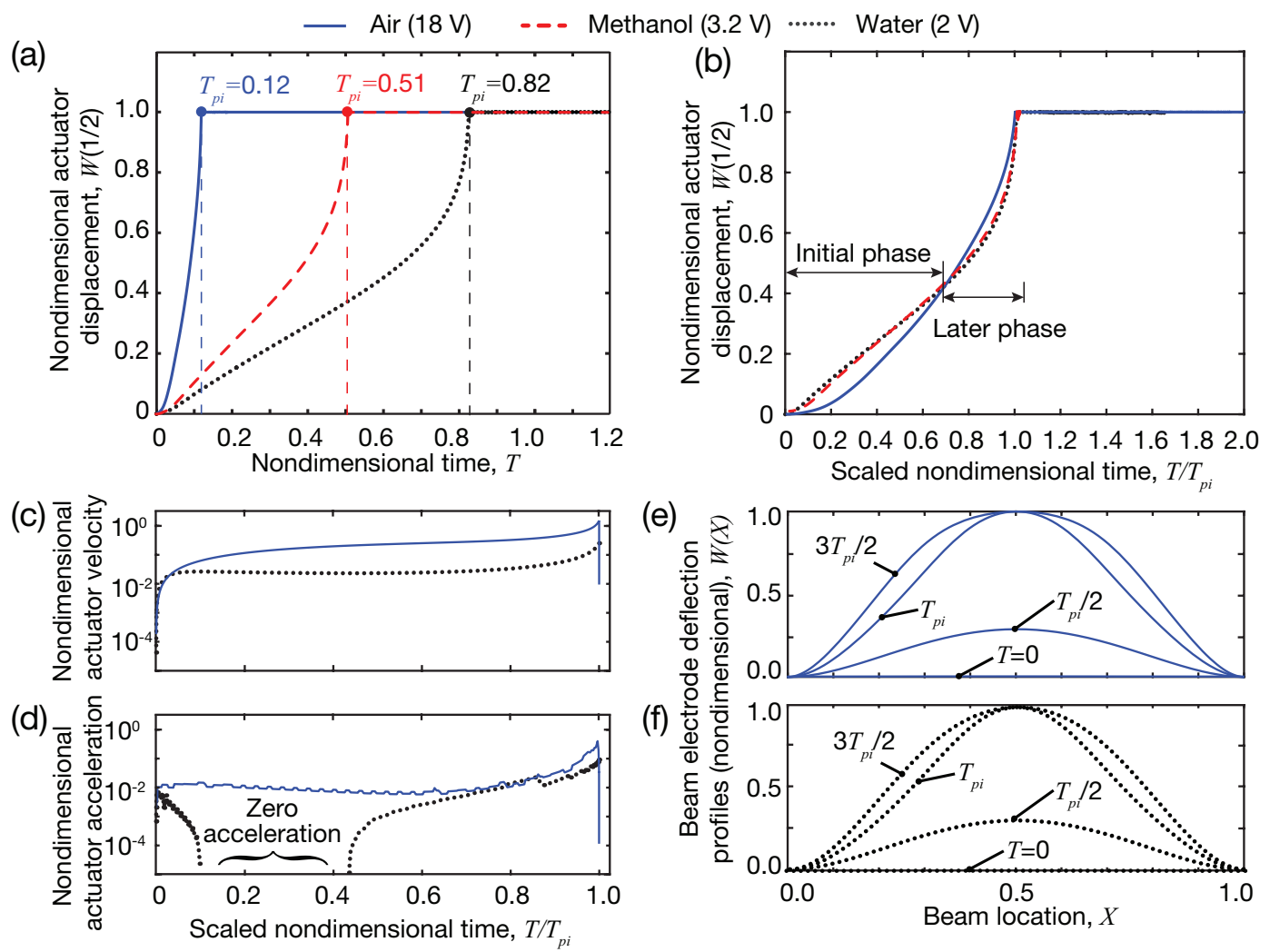

Figure 3: Actuator dynamic response in air, methanol, and water media. Plot showing the onset of pull-in instability for a step input voltage amplitude $\bar{V}$ in (a) absolute nondimensional time $T$ (b) scaled nondimensional time $T / T_{p i}$. (c,d) Nondimensional actuator velocity and acceleration for water and air media. (e,f) Beam electrode deflection profiles at different instances in time. Here, $T_{p i}$ corresponds to nondimensional pull-in time for a specific media.

\section{Results and Discussion}

The actuator shown in Fig. 1 with water as viscous media and parameters given in Table 1 is treated as the reference case. The actuator model (Eq. 5 ) is numerically solved to analyze the static and dynamic behavior of the actuator characterized in terms of pull-in time and displacement. Based on our preliminary work, we found that the first five symmetric modes $(2 M-1 \in\{1,3,5,7,9\})$ of the beam electrode is acceptable to study the mechanics of the actuator (see Appendix). Hence for the subsequent analysis, we chose the total number of mode shapes $M=5$.

\subsection{Static behavior}

The response of the actuator to a DC actuation voltage $\bar{V}$ in three viscous media is characterized through static pull-in analysis (Fig. 2). For all the three media, the static equilibrium curves plotted with normalized DC actuation voltages $\left(\bar{V} / \bar{V}_{p i}\right)$ overlap with one another, highlighting the absence of viscous effects (Fig. 2a). Here, the DC actuation voltages $(\bar{V})$ for a given media are normalized with corresponding pull-in voltages $\left(\bar{V}_{p i}\right)$. For actuation voltages below the static pull-in, that is along the lower branch, the actuator operates in a stable manner with a maximum displacement sensitivity of $0.46,1.51$, and $6.44 \mu \mathrm{m} / \mathrm{V}$ in air, methanol, and water media, respectively. However, beyond the pull-in 

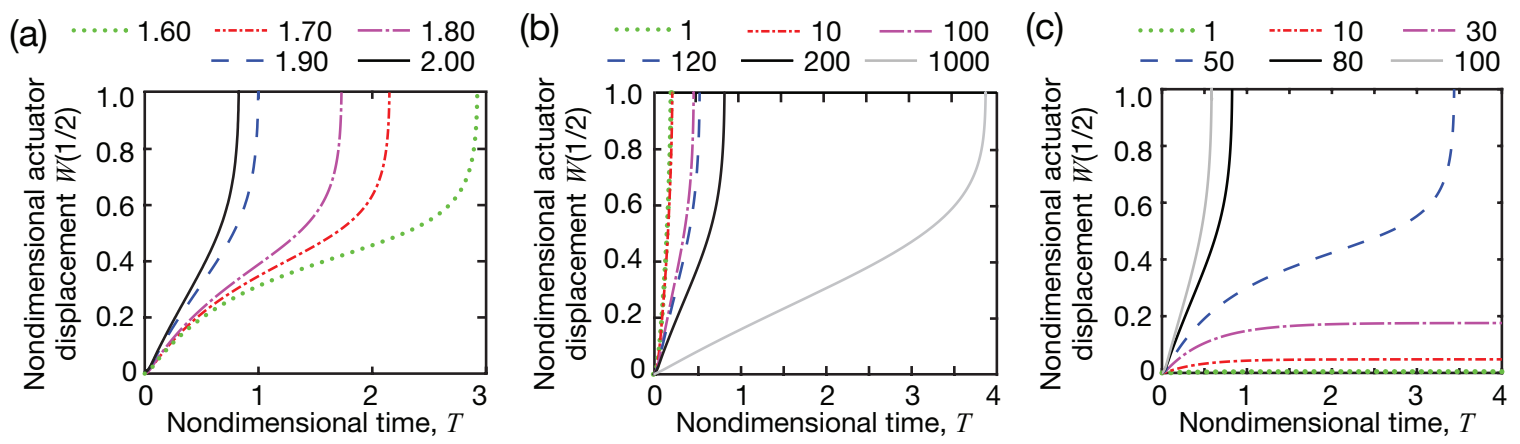

Figure 4: The effect of media properties on the actuator pull-in dynamics. (a) In water media for different actuation voltage amplitudes $\bar{V}$ and nondimensional damping term $c=200$. (b) For actuation voltage amplitude $\bar{V}=2 \mathrm{~V}$, relative permittivity $\epsilon_{f, w}=80$ and different nondimensional damping terms $c$. (c) For actuation voltage amplitude $\bar{V}=2 \mathrm{~V}$, nondimensional damping term $c=200$, and different relative permittivities $\epsilon_{f}$.

voltage, the actuator quickly snaps into the stationary electrode due to the dominance of the nonlinear electrostatic force. For all the three media, the nondimensional actuator position $W(1 / 2)$ at the onset of the pull-in instability is 0.46 .

\subsection{Dynamic behavior}

The dynamic response of the actuator in three viscous media is characterized in terms of nondimensional pull-in times $T_{p i}$ and nondimensional actuator amplitude $W(1 / 2)$. Here, the inertial, viscous, and contact force effects are considered as they are known to affect the dynamic response of the actuator [27].

The effect of media properties on the actuator pullin time is determined by supplying the actuator with a step voltage input $\bar{V}$, where the step voltage amplitude $\bar{V}$ is chosen to hold the relation $\bar{V}>\bar{V}_{p i}$ (Figs. 3 and 4 . Note that the amplitudes of the step voltage input $\bar{V}$ for the media are chosen such that the generated electrostatic forces are equal for $W=0$. The high relative permittivity of water $\left(\epsilon_{f, w}=80\right)$ amplifies the generated force, resulting in pull-in to occur at lower actuation voltage $(\bar{V}=2 \mathrm{~V})$ compared to air $(\bar{V}=18 \mathrm{~V})$; while, the high dynamic viscosity of water $\left(\mu_{f, w}=8.9 \times 10^{-4} \mathrm{~Pa}-\mathrm{s}\right)$ increases the pull-in time (Fig. 33). During the initial phase of the pull-in, an actuator will have higher speed in water compared to air; however, during the later phase, the actuator will have a lower speed. This can be explained as follows: the initial higher speed is due to the higher electrostatic force - due to higher relative permittivity $\epsilon_{f}$ - and the later lower speed is due to the higher viscous friction - due to higher dynamic viscosity $\mu_{f}$ (Fig. 3 b-d). Moreover, as the actuator accelerates from $W=0$ position, it gains speed, and this increase in speed exacerbates the viscous damping effect, resulting in lower speeds during the later phase of the pull-in. A plot of beam electrode deflection profiles at different instances in time show that after the beam electrode pulls into the stationary electrode ( $\left.T=T_{p i}\right)$, the beam electrode zips into the stationary electrode $\left(T=3 T_{p i} / 2\right)$, increasing the length of contact.

The actuator response near the vicinity of the pullin instability is sensitive to the actuation voltage $V$. For instance, the actuator in water media with a pullin voltage amplitude $\bar{V}=1.59 \mathrm{~V}$ exhibits a wide range of pull-in times - a $25 \%$ increase in actuation voltage amplitude $\bar{V}$ (from 1.60 to $2.00 \mathrm{~V}$ ) can lower the pullin time to a third (Fig. 4a). Fig. 4p highlights the effect of viscous damping by the media on the actuator. For a fixed $\epsilon_{f}$ and fixed actuation voltage amplitude $\bar{V}$, an increase in viscous damping modeled by nondimensional damping term $c$ delays the pullin time $T$ by up to 16 times. Similarly, for a fixed actuation voltage amplitude $\bar{V}$ and damping term $c$, an increase in relative permittivity of the media $\epsilon_{f}$ can cause the pull-in instability to occur and reduce 


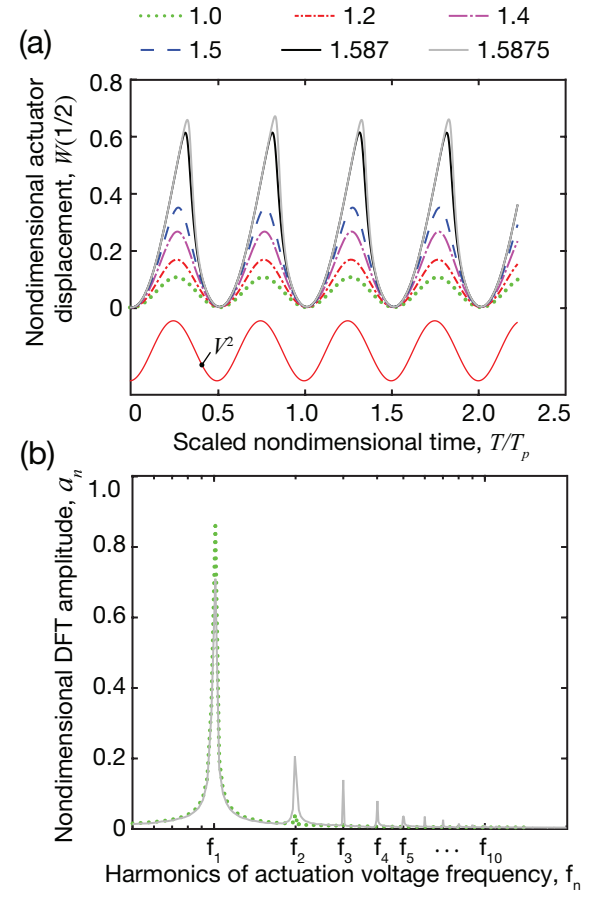

Figure 5: Actuator dynamic response to a sinusoidal voltage input $V$ in water media. (a) Displacement trace of the actuator $W(1 / 2)$ for different actuation voltage amplitudes $\bar{V}$ and voltage frequency $f=100 \mathrm{~Hz}$. Note that the time period $T_{p}$ refers to the voltage waveform, where $T_{p}=1 /\left(t_{p} f\right)$. (b) A DFT of the actuator displacement trace $W(1 / 2)$ simulated for different actuation voltage amplitudes $\bar{V}$ and voltage frequency $f=100$ $\mathrm{Hz}$. The nondimensional DFT amplitude $a_{n}$ is scaled with the actuator amplitude $W(1 / 2)$ at the first harmonic frequency $f_{1}=100 \mathrm{~Hz}$.

the pull-in time $T$ by up to 3 times (Fig. 44).

The actuator displacement traces $W(1 / 2)$ for different sinusoidal voltage waveforms $V$ in water media captures the nonlinearities in the actuator (Figs. 5 and 6). The actuator dynamics can be approximated to linear for lower actuation voltage amplitudes $\bar{V}<1.5 \mathrm{~V}$ and/or high actuation frequencies $f>200 \mathrm{~Hz}$. Applying a sinusoidal voltage waveform with frequency $f=100 \mathrm{~Hz}$ and higher voltage amplitudes $(\bar{V}>1.5 \mathrm{~V})$ excites the higher harmonics $f_{n}\left(\forall n \in \mathbb{Z}_{>0}\right)$ in the actuator, as computed by applying the discrete Fourier transform (DFT) to the simulated displacement trace $W(1 / 2)$ (Fig. 5). Note

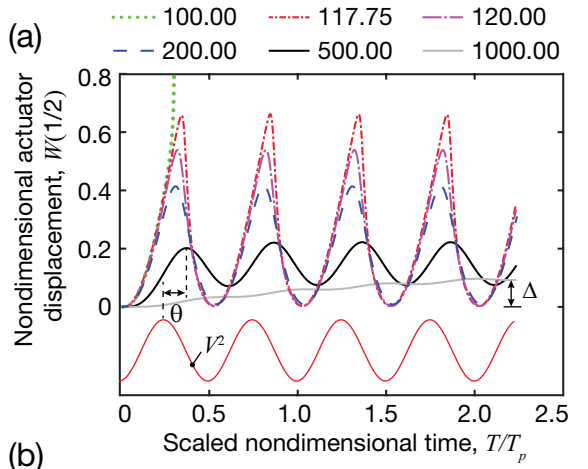

(b)

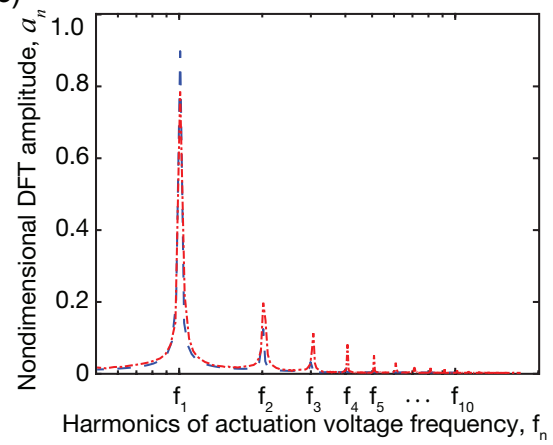

Figure 6: Actuator dynamic response to a sinusoidal voltage input $V$ in water media. (a) Displacement trace of the actuator $W(1 / 2)$ for different actuation voltage frequencies $f$ and voltage amplitude $\bar{V}=1.6 \mathrm{~V}$. Note that the time period $T_{p}$ refers to the voltage waveform, where $T_{p}=1 /\left(t_{p} f\right)$. (b) A DFT of the actuator displacement trace $W(1 / 2)$ simulated for different actuation voltage frequencies $f$ and voltage amplitude $\bar{V}=1.6$ $\mathrm{V}$. The nondimensional DFT amplitude $a_{n}$ is scaled with the actuator amplitude $W(1 / 2)$ at the first harmonic frequency $f_{1}$.

that the $n^{\text {th }}$ order harmonic frequency $f_{n}=n f$ and the nondimensional DFT amplitude

$$
a_{n}=\frac{\frac{2}{P}\left|\sum_{p=0}^{P-1} W(1 / 2)_{\mid p} e^{-j \frac{2 \pi n p}{P}}\right|}{\max W(1 / 2)_{\mid f}} .
$$

As can be seen in Eq. 6, $a_{n}$ is scaled with the actuator amplitude, $\max W(1 / 2)$ for voltage frequency $f \mathrm{~Hz}$. Here, $j=\sqrt{-1}$ and $P$ is the number of sample points used in displacement measurement $W(1 / 2)$. To prevent aliasing, a minimum of 1125 sample points were chosen for the computation. The presence of higher harmonics resulted in a skewed sinusoidal response instead of a pure sinusoidal response - an indication 
of a nonlinear system. On the contrary, for the lower voltage amplitudes $(\bar{V}<1.5 \mathrm{~V})$, the higher harmonics $f_{n}(\forall n \in \mathbb{N})$ vanish with the appearance of a very low amplitude $\left(<0.2 a_{1}\right)$ for second harmonic response $f_{2}$ - the actuator can be approximated to be a linear system. For high $\bar{V}$ and high $f$, the actuator may pull into the stationary electrode which may result in not releasing the beam electrode; an example scenario is for $\bar{V}=1.6 \mathrm{~V}$ and $f=100 \mathrm{~Hz}$ (Fig. 6). For very high $f$ operation $(f>500 \mathrm{~Hz})$, the beam electrode does not pull-in to the stationary electrode, and a significant phase lag $\left(\theta=39^{\circ}\right)$ and displacement drift $(\Delta=0.092 W)$ over time is observed.

\section{Conclusion}

In this paper, we analyzed the actuator response in a clamped-clamped configuration immersed in viscous dielectric media. We modeled the actuator as a continuous system by deriving a reduced-order model, and solving it by employing the Galerkin method and linear undamped mode shapes for a clamped-clamped beam. The model is utilized to study the actuator dynamics over a broad range, three orders of magnitude of viscosity and two orders of magnitude of relative permittivity of the media. We make three important observations: (1) the actuator at lower actuation voltage amplitudes $\bar{V}<1.5 \mathrm{~V}$ and/or higher actuation frequencies $f>200 \mathrm{~Hz}$ can be approximated to be a linear system; (2) the high relative permittivity of a media amplifies the generated force, resulting in pull-in to occur at lower actuation voltage amplitudes, and the high dynamic viscosity of the media increases the pull-in time; and (3) at very high actuation frequencies $(f>500 \mathrm{~Hz})$, the beam electrode does not pull-in into the stationary electrode, and the actuator experiences a significant phase lag $\left(\theta=39^{\circ}\right)$ and displacement drift $(\Delta=0.092$ $W$ ) over time.

\section{Appendix}

The model (Eq. 5) is validated for the static and dynamic performance of the actuator by benchmarking against the data presented in Ref. 9]. The actuator shown in Fig. 1 with parameters given in Ref.
[9], that is, $l=610 \mu \mathrm{m}, b=40 \mu \mathrm{m}, h=2.1 \mu \mathrm{m}$, $d=2.3 \mu \mathrm{m}, c=260, \epsilon_{f}=1$, and $\hat{N}=-3.7 \mu \mathrm{N}$ is treated as the reference case. Three different cases where the total number of mode shapes $M \in\{3,4,5\}$ are studied and compared with data obtained from Ref. [9] (Fig. 7). Our study shows that employing the first five symmetric modes $\left(\phi_{1}, \phi_{3}, \phi_{5}, \phi_{7}, \phi_{9}\right)$ in the model (Eq. 5) will be sufficient to predict actuator displacement and pull-in time (Fig. 7b). A similar study has been conducted by Ref. 24] and showed that using the first five symmetric modes will result in only $10 \%$ error in contact length predictions of the beam electrode with the stationary electrode.
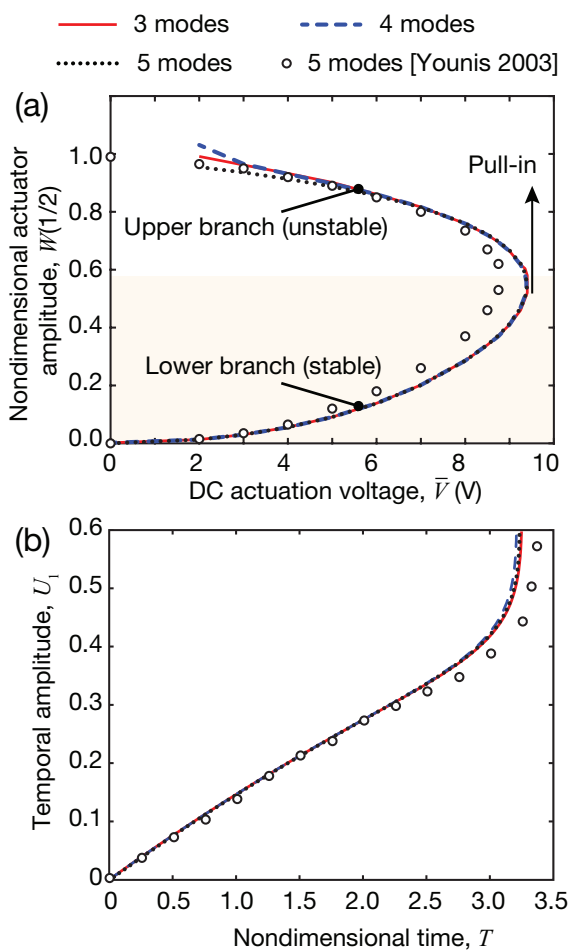

Figure 7: Our model validation. (a) Static equilibrium curves for the actuator computed for $M=3$ (solid), $M=4$ (dashed), $M=5$ (dotted) modes and compared with results from Ref. 9 (Younis 2003, circles). Note the Young's modulus $E$ of the beam used in this static analysis is $149 \mathrm{GPa}$. (b) Dynamic response of the actuator for a step input voltage amplitude $\bar{V}$ computed for $M=3$ (solid), $M=4$ (dashed), $M=5$ (dotted) modes and compared with results from Ref. [9] (Younis 2003, circles). Note the Young's modulus $E$ of the beam used in this dynamic analysis is $166 \mathrm{GPa}$. 


\section{Acknowledgment}

This work was supported in part by Wichita State University capitalization funds.

\section{References}

[1] R. Syms, H. Zou, J. Yao, D. Uttamchandani, J. Stagg, Scalable electrothermal mems actuator for optical fibre alignment, Journal of Micromechanics and Microengineering 14 (12) (2004) 1633 (2004).

[2] S. H. Lee, K.-C. Lee, S. S. Lee, H.-S. Oh, Fabrication of an electrothermally actuated electrostatic microgripper, in: TRANSDUCERS '03. 12th International Conference on Solid-State Sensors, Actuators and Microsystems. Digest of Technical Papers (Cat. No.03TH8664), Vol. 1, 2003, pp. 552-555 vol.1 (June 2003). doi:10. 1109/SENSOR. 2003.1215376

[3] V. Mukundan, W. J. Nelson, B. L. Pruitt, Microactuator device for integrated measurement of epithelium mechanics, Biomedical microdevices 15 (1) (2013) 117-123 (2013).

[4] B. G. Kilberg, D. S. Contreras, J. Greenspun, K. S. Pister, Mems aerodynamic control surfaces for millimeter-scale rockets, in: Manipulation, Automation and Robotics at Small Scales (MARSS), 2017 International Conference on, IEEE, 2017, pp. 1-5 (2017).

[5] G. M. Rebeiz, K. Entesari, I. C. Reines, S. Park, M. A. El-tanani, A. Grichener, A. R. Brown, Tuning in to rf mems, IEEE Microwave Magazine 10 (6) (2009) 55-72 (Oct 2009). doi: 10.1109/MMM. 2009.933592

[6] D. J. Bell, T. J. Lu, N. A. Fleck, S. M. Spearing, Mems actuators and sensors: observations on their performance and selection for purpose, Journal of Micromechanics and Microengineering 15 (7) (2005) S153 (2005).

URL http://stacks .iop.org/0960-1317/15/ $\mathrm{i}=7 / \mathrm{a}=022$
[7] P. B. Chu, P. R. Nelson, M. L. Tachiki, K. S. Pister, Dynamics of polysilicon parallel-plate electrostatic actuators, Sensors and Actuators A: Physical 52 (1) (1996) 216 - 220, proceedings of the 8th International Conference on SolidState Sensors and Actuators Eurosensors IX (1996). doi:https://doi.org/10.1016/09244247(96)80152-X. URL http://www.sciencedirect.com/ science/article/pii/092442479680152X

[8] A. Rollier, B. Legrand, D. Collard, L. Buchaillot, The stability and pull-in voltage of electrostatic parallel-plate actuators in liquid solutions, Journal of Micromechanics and microengineering 16 (4) (2006) 794 (2006).

[9] M. I. Younis, E. M. Abdel-Rahman, A. Nayfeh, A reduced-order model for electrically actuated microbeam-based mems, Journal of Microelectromechanical systems 12 (5) (2003) 672-680 (2003).

[10] J. F. Rhoads, S. W. Shaw, K. L. Turner, The nonlinear response of resonant microbeam systems with purely-parametric electrostatic actuation, Journal of Micromechanics and Microengineering 16 (5) (2006) 890 (2006).

[11] W.-M. Zhang, H. Yan, Z.-K. Peng, G. Meng, Electrostatic pull-in instability in mems/nems: A review, Sensors and Actuators A: Physical 214 (2014) 187-218 (2014).

[12] S. Saghir, M. I. Younis, Approaches for reducedorder modeling of electrically actuated vonkarman microplates, Journal of Computational and Nonlinear Dynamics 12 (1) (2017) 011011 (2017).

[13] H. Farokhi, M. H. Ghayesh, S. Hussain, Pullin characteristics of electrically actuated mems arches, Mechanism and Machine Theory 98 (2016) 133-150 (2016).

[14] S. P. Burugupally, J. A. Mangels, Performance evaluation of a curved electrode actuator fabricated without gold/chromium conductive layers 
Microsystem Technologies 24 (8) (2018) 34793485 (Aug 2018). doi:10.1007/s00542-0183751-3.

URL https://doi.org/10.1007/s00542-0183751-3

[15] D. J. Hoelzle, C. K. Chan, M. B. Scott, M. A. Lake, A. C. Rowat, A large displacement, high frequency, underwater microelectromechanical systems actuator, Journal of Applied Physics 117 (1) (2015) 014503 (2015).

[16] S. P. Burugupally, D. Hoelzle, Experimental investigation of curved electrode actuator dynamics in viscous dielectric media, Applied Physics Letters 113 (7) (2018) 074102 (2018). arXiv:https://doi.org/10.1063/1. 5042456, doi:10.1063/1.5042456.

URL https://doi.org/10.1063/1.5042456

[17] M. Li, V. T. Rouf, D. A. Horsley, Substrate effect in squeeze film damping of lateral oscillating microstructures, in: Micro Electro Mechanical Systems (MEMS), 2013 IEEE 26th International Conference on, IEEE, 2013, pp. 393-396 (2013).

[18] M. S. Ahmed, M. Ghommem, A. Abdelkefi, Shock response of electrostatically coupled microbeams under the squeeze-film damping effect, Acta Mechanica 229 (12) (2018) 5051-5065 (2018).

[19] M. Gomez, D. Vella, D. E. Moulton, Pull-in dynamics of overdamped microbeams, Journal of Micromechanics and Microengineering 28 (11) (2018) 115002 (2018).

[20] B. Preetham, M. A. Lake, D. J. Hoelzle, A curved electrode electrostatic actuator designed for large displacement and force in an underwater environment, Journal of Micromechanics and Microengineering 27 (9) (2017) 095009 (2017).

[21] A. H. Nayfeh, M. I. Younis, E. M. AbdelRahman, Reduced-order models for mems applications, Nonlinear Dynamics 41 (1) (2005) 211236 (Aug 2005). doi:10.1007/s11071-0052809-9.
URL https://doi .org/10.1007/s11071-0052809-9

[22] L. Meirovitch, Fundamentals of Vibrations, McGraw-Hill higher education, McGraw-Hill, 2001 (2001).

[23] P. Gonçalves, M. Brennan, S. Elliott, Numerical evaluation of high-order modes of vibration in uniform euler-bernoulli beams, Journal of sound and vibration 301 (3-5) (2007) 1035-1039 (2007).

[24] C. P. Vyasarayani, E. M. Abdel-Rahman, J. McPhee, S. Birkett, Modeling mems resonators past pull-in, Journal of Computational and Nonlinear Dynamics 6 (3) (2011) 031008031008-7 (Feb 2011). doi:10.1115/1.4002835. URL http://dx.doi.org/10.1115/1.4002835

[25] T. L. Sounart, T. A. Michalske, K. R. Zavadil, Frequency-dependent electrostatic actuation in microfluidic mems, Journal of Microelectromechanical Systems 14 (1) (2005) 125-133 (Feb 2005). doi:10.1109/JMEMS . 2004.839006.

[26] R. M. Shih, D. S. Contreras, T. L. Massey, J. T. Greenspun, K. S. Pister, Characterization of electrostatic gap-closing actuator arrays in aqueous conditions, in: Micro Electro Mechanical Systems (MEMS), 2018 IEEE, IEEE, 2018, pp. 596-599 (2018).

[27] D. Elata, On the static and dynamic response of electrostatic actuators, Bulletin of the Polish Academy of Sciences. Technical Sciences Vol. 53, nr 4 (2005) 373-384 (2005). 